\title{
Las relaciones sociales y educativas existentes entre los elementos personales del proceso educativo durante una sesión de Educación Física. Un estudio de casos
}

\section{The social and educational relations between the personal elements of the educational process during a meeting of physical education. Acase study}

\author{
Jose Francisco Sánchez Arroyo, Jose Luis Chinchilla Minguet, Manuel de Burgos Carmona, Oscar Romero Ramos \\ Universidad de Málaga (España)
}

Resumen: el objetivo de la presente investigación es estudiar e investigar acerca de las relaciones sociales y educativas que se dan entre los elementos personales del proceso educativo durante las clases de Educación Física. Concretamente hemos estudiado el clima de aula de un grupo clase con tan sólo diez alumnos y alumnas perteneciente a un educativo unitario de educación primaria, y donde existe diferencia de edad entre los alumnos/as, pues hay niños de edades comprendidas entre los ocho y doce años. Para ello utilizamos el enfoque naturalista/cualitativo o etnográfico, y concretamente realizaremos un estudio de casos. Pues este enfoque nos ha permitido estudiar, analizar, descubrir, interpretar y comprender el grupo clase elegido y el comportamiento individual y social de sus componentes.

Palabras claves: clima de aula, relaciones sociales, relaciones educativas, estudio de casos.

Abstract: the objective of the present investigation is to study and investigate into the social and educative relationships among the people involved in the educative process in the physical education classes. We have focused our study on the classroom environment of a class group of only the students in a unitary center of primary education, whit an age range from eight to twelve. Therefore, we have used the naturalist/qualitative or ethnographich approach, and particularly we will carry out a study of caser. Since this approach has allowed us to study, analize, find out, intreprete and understand the selected class group and the individual and social behaviour of its members.

Key words: classroom environment, social relationships, educational relations, a case study.

\section{Introducción}

El proceso de enseñanza y aprendizaje, tal y como indica el Decreto $230 / 2007$, es un proceso social y personal en el que cada individuo debe relacionarse, activamente con las personas que le rodean. Para Sáenz-López (1997), las interacciones sociales que se producen en el área de Educación Física son mayores que en las demás áreas, necesitando una metodología específica para crear ambientes de aprendizaje, donde las relaciones sociales sean correctas y positivas para conseguir eficacia en el proceso educativo.

En este sentido, el clima de aula es un elemento de vital importancia para determinar cómo son las relaciones sociales dentro del proceso educativo, y nos indica cómo es el ambiente de aprendizaje. «Los estos estudios relacionados con el clima de aula han ido cobrando mayor interés ha medida que se ha ido relacionando el clima de aula con los resultados educativos.» (Martínez, 1994, p. 46).

El clima de aula supone las interacciones de tipo socio-afectivos producidas durante la intervención en el aula dentro del proceso de

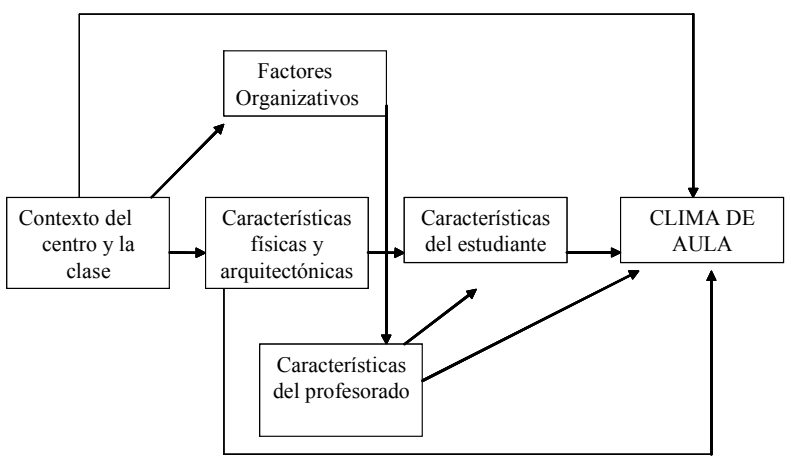

Figura 1. Determinantes del clima de aula (Gairín, 1986, p. 278)

Fecha recepción: 03-12-07 - Fecha envío revisores: 06-12-07 - Fecha de aceptación: 30-06-08 Correspondencia: Jose Francisco Sánchez Arroyo

C/Goya, $\mathrm{N}^{\circ} 30,2^{\circ}$ derecha

29640 Fuengirola - Málaga (España)

E-mail: webmedialuna@hotmail.com enseñanza y aprendizaje. «Las interacciones entre los alumnos y entre éstos y el profesor son determinadas por y determinantes del clima de aula» (Ascorra y cols., 2003, p. 119). Los factores que influyen en el clima de aula son: (figura 1).

Teniendo en cuenta lo expuesto, los objetivos que nos planteamos en la presente investigación son:

Estudiar las relaciones sociales y educativas existentes entre los elementos personales del proceso de enseñanza y aprendizaje en una clase de Educación Física.

- Estudiar y comprobar los factores que determinan el clima.

- Observar el clima de aula en un grupo clase con diez alumnos/as y donde existe diferencia de edad entre ellos, y pertenecientes a un centro educativo unitario.

- Observar y analizar la relación social, educativa y afectiva existente entre el docente y los alumnos y entre los alumnos entre sí.

\section{Material y método}

Utilizaremos un enfoque naturalista/cualitativo o etnográfico, concretamente realizaremos un estudio de casos. Con el objetivo de estudiar, analizar, descubrir, interpretar y comprender el grupo clase seleccionado y el comportamiento individual y social de sus componentes. Esta metodología ha sido utilizada por Gutiérrez (1994); Heras (1994); Plaza (1994); y Prada (1995), en sus diferentes tesis doctorales.

El hecho de que nuestro objeto de estudio sea dinámico, holístico y múltiple, nos lleva a no tratar deductivamente hipótesis previas, sino a indagar de forma descriptiva las relaciones existentes entre los elementos personales del grupo clase, y a posteriori, tras la observación e interrogación de la realidad, sacar hipótesis y conclusiones de manera inductiva, con el fin de establecer generalizaciones acerca de la más amplia población a la que pertenece la unidad estudiada.

La investigación se llevo a cabo, durante el curso académico completo, en un colegio público y unitario de educación primaria del interior de la provincia de Málaga. La elección de dicho centro viene dada porque me permitió estudiar el clima de aula en una clase de Educación Física con tan sólo diez alumnos y alumnas, y donde existe diferencia de edad entre ellos, pues sus edades estaban comprendidas entre los ocho y doce años. Se trata un centro educativo unitario que sólo tiene tres grupos clases, correspondientes a los tres ciclos educativos de educa- 
ción primaria. El muestreo teórico sobre el que se realizó la investigación corresponde a los alumnos y alumnas de $\operatorname{los} \operatorname{cursos} 3^{\circ}, 4^{\circ}, 5^{\circ}$ y $6^{\circ}\left(2^{\circ}\right.$ y $3^{\circ}$ ciclo) pues realizaban juntos la clase de Educación Física.

\subsection{Recursos metodológicos}

Recursos para observar la realidad: hemos utilizado el observación participante directa, a largo plazo, persistente y continua como instrumento indispensable para el análisis de los comportamientos que se dan en el entorno o clima de la clase. Además de las notas de campo tomadas por el investigador y el análisis de documentos (programación de aula y diario de clase del docente).

Registros para interrogar la realidad: las entrevistas, puestas en práctica con el fin de conocer ideas y percepciones de los protagonistas (profesores, alumnos/as, padres y madres). Los cuestionarios (de respuesta cerrada y abierta), utilizados para completar la información obtenida mediante la entrevista y la observación. Y la triangulación para contrastar la información obtenida durante el trabajo de campo, con objeto de reforzar la veracidad y la credibilidad de los resultados de la investigación.

\subsection{Análisis de documento}

Se han ordenado sistemáticamente las informaciones procedentes de la aplicación de los recursos metodológicos, con el objetivo de analizarla, darla a conocer a través del informe, y finalmente, realizar las generalizaciones finales. Para ello, hemos seguido las fases propuestas por Gutiérrez (2006):

1.Reflexión inicial, análisis especulativo o descubrimiento: se realizó una lectura pausada y repetida de los primeros datos, para hacer las primeras interpretaciones e ir descubriendo las primeras pistas o temas que servirán para empezar a estructurar la información y reorientar la nueva recogida de datos.

2.Estructuración o codificación de datos: supone la agrupación y ordenación de la información en diferentes categorías, lo cual nos permite manejar la información con mayor precisión y utilizarlas cuando lo requiramos para realizar el informe.

3.Generalización: son consideraciones finales cuyo objetivo es hacer extensible a otros casos las conclusiones obtenidas en el caso estudiado.

\section{Resultados: el informe}

Mediante el informe hacemos públicos los datos aparecidos durante la investigación, analizando la realidad estudiada en toda su heterogeneidad, de modo que el lector pueda aproximarse lo más posible a la percepción que el investigador tuvo de los hechos. Se ha realizado un sistema de codificación para asegurar la confidencialidad del trabajo: $(O$ : observación; $E A$ : entrevista a un alumno; $E P$ : entrevista al profesor; $C A$ : cuestionario a un alumno; $C P$ : cuestionario al profesor; $A D$ : análisis de documentos). A continuación, mostramos las categorías emergentes tras el análisis de los datos aparecidos en la presente investigación:

Relaciones del profesor con los alumnos y alumnas.

Durante las primeras sesiones se puede observar que el profesor quiere dejar claro algunos aspectos importantes que serán puestos en prácticas durante todo el año $(\mathrm{O})$. Así, revisando la programación de aula vemos que la primera unidad didáctica va destinada al trabajo de los hábitos higiénicos y al aprendizaje de normas de clase $(\mathrm{AD})$.

«Es bueno que aprendan desde el principio normas y comportamientos que puedan llevar a la práctica durante todo el año. Todo ello con el objetivo de mejorar las normas de convivencia y las relaciones sociales del grupo» (EP).

Se pueden observar como hay periodos en los que la clase es parada por el docente para plantear debates y el dialogo entre todos $(\mathrm{O})$ :

«Es bueno que se produzca discusión entre los alumnos yalumnas. Así, aprenden a defender sus puntos de vista de una manera dialogada». (EP).
El maestro trata por igual a todos los alumnos y alumnas aunque se observan algunas diferencias, puesto que todos y cada uno de ellos tienen unas características, personalidad y un nivel y ritmo de aprendizaje $(\mathrm{O})$ :

«Todos son trabajadores, aunque una alumna lo es más que los demás» (EP).

El pedagogo aplica un conocimiento de resultados positivo y afectivo, utilizando expresiones como: «Muy bien Antonio, sigue así». Además, usa estilos de enseñanza participativos, pues los alumnos debieron explicar juegos a sus propios compañeros $(\mathrm{O})$ :

«Resulta positivo pues necesita una implicación plena por parte de todos los alumnos evitando que cada uno vaya a lo suyo». (EP).

En cuanto a la organización del grupo clase, todas las sesiones empiezan cuando los alumnos se van a la esquina del gimnasio y se sientan en los bancos suecos $(\mathrm{O})$.

"Es necesario crear en los alumnos unos hábitos de comportamiento y de trabajo. Así, la clase funciona mejor y no perdemos tanto tiempo». (EP).

Cabe mencionar que de vez en cuando se produce algún mal comportamiento, pero estos son hechos aislados. Siempre que se ha producido esta situación, el docente le llama a la atención al alumno. Por ejemplo, una alumna empujo a un compañero y el maestro paro inmediatamente el juego y explico a los demás la acción incorrecta realizada por su compañera, con el objetivo de que esa acción no vuelva a suceder. (O).

\section{Relaciones de los alumnos y alumnas con el profesor.}

Dentro de las clases de Educación Física la relación del alumnado con el docente es adecuada y positiva (O). Se comprueba que los niños aceptan al profesor tanto en su manera de explicar, de ser, de tratarles... es decir, están muy contentos con él. En este sentido aparecen afirmaciones como:

«Mellevo muy bien conél. Es muy enrollado ynos enseña mucho». (EA).

Pero algunas respuestas de los alumnos van un poco más allá y hablan de la forma de ser del docente y de cómo esto influye positivamente en sus clases:

[T. «Exlica muy bieny además es muy buena gente porque no regaña mucho, me lo paso muy bien en sus clases.» (EA).

Así, en las sesiones se observa buen ambiente, pues casi siempre los chicos y chicas están contentos y riéndose. Además, el maestro participa en algunas actividades con el objetivo de dar mayor aliciente a la actividad $(\mathrm{O})$.

Es común que durante las clases surjan temas de conversación o debate relacionados con el deporte y con la asignatura $(\mathrm{O})$. El profesor lo hace con el objetivo de crear como una especie de debate o charla para que hablen entre ellos (CO) y los alumnos lo ven de buen agrado:

«Este profesor bueno, explica muy bien y es como sifuera un amigo mío porque en la clase se hablan de cosas que no vienen en los libros. (EA).

Relaciones de los alumnos/as entre sí.

En este grupo clase todos los alumnos/as se conocen entre sí, pues en años anteriores también han estado juntos, dado que en la clase de Educación Física se une segundo ciclo (tercero y cuarto) y tercer ciclo (quinto y sexto).

Por ello, se observa que el grupo esta cohesionado y que no hay grandes problemas de comportamiento entre ellos. Así, a la pregunta, ¿estás contento con el grupo de compañeros y compañeras que te ha tocado este año?, respondieron:

«Si hay mucha gente y esta bien para jugar a algo». (CA)

Se observa que existe compañerismo entre el alumnado, lo cual implica ser cercanos y apoyarse entre ellos, manifestando interés en las actividades que realizan los demás. Pero esta relación tan positiva no ha sido así desde el principio, pues hay chicos que han mostrado actitudes de poca colaboración con sus compañeros:

«A ella le ha costado relacionarse con sus compañeros, pero ahora parece que ya empieza a no tener problemas a la hora de relacionarse con los demás» (EP). 
Pero el caso de esta niña es aislado y gracias al trabajo del profesor, quién paraba la actividad cada vez que existía una discusión para solucionarlo de manera dialogada y en grupo; $\mathrm{y}$ a la puesta en práctica de unas actividades y juegos grupales desde el principio de curso, comenzaron a integrarla en el grupo. De manera que a medida que avanzaba el curso, ya se relacionaban todos con todos, es decir, que se observa que no hay ningún alumno ni alumna aislado o discriminado socialmente por sus compañeros.

Es decir, parece que el hecho de ser tan pocos en clase hace que todos se consideren amigos de todos, en mayor o menor medida. Además el hecho de tratarse de un pueblo pequeño, hace que se relacionen y jueguen en la calle con los niños de su misma clase, puesto que no hay más niños de su edad en el pueblo:

"Yo me llevo muy bien con todo el mundo. Y los demás, se llevan bien entre ellos porque todos se comportan muy bien, no hay peleas $n i$ peleas» (EA).

Respecto a la relación de los chicos con las chicas, desde el principio del curso, las chicas siempre se ponían juntas, tanto a la hora de la explicación de los ejercicios como en el momento de formar parejas para realizar cualquier actividad $(\mathrm{O})$. El docente comento en varias ocasiones que los niños y las niñas deben jugar junto y formar parejas mixtas. Para lograr el objetivo de la coeducación el profesor creó el carné de Educación Física, donde daba puntos positivos a aquellos alumnos y alumnas que formaran grupos mixtos.

A medida que pasaba el curso se iban observando más agrupaciones mixtas, con objetivo para los alumnos y alumnas de tener puntos positivos al acabar la sesión. Pero al suceder esto, se estaba cumpliendo el objetivo del docente: la coeducación. Cuando pasaba la sesión sin formarse grupos mixtos, el docente lo remarcaba: «hoy no doy puntos positivos para nadie porque no os habéis puesto los chicos con las chicas».

«En el recreo también jugamos con los niños porque como somos pocos en el colegio casi siempre jugamos todos juntos.» (EA).

El ambiente en el aula.

Cada vezque el profesor da explicaciones sobre la tarea o elejercicio a realizar, todos escuchan aunque hay un alumno que siempre se muestra distraído, por lo que no comprende lo que hay que hacer pues no ha estado atento a la explicación del docente.

"En las explicaciones todos escuchan aunque hay alumnos que se distraen, por ello dirijo mi mirada hacia ellos, para que vean que les estoy controlando. Con lo que evito que me interrumpan mientras doy la explicación». (EP).

En el aula se observa un ambiente de aprendizaje y social muy tranquilo, lo cual es reconocido por el alumnado en sus respuestas a las preguntas planteadas en los cuestionarios:

«El ambiente es tranquilo, nunca hacemos ruido». (CA).

Así, se puede observar que los alumnos están cómodos en las clases de Educación Física y participan de manera desinhibida y divertida. En este sentido, en los cuestionarios pasados al alumnado, cuatro de ellos señalaron que siempre les gustan las clases de Educación Física, y los seis restantes afirman que casi siempre les gustan las clases de esta asignatura. (CA).

"Las clases de Educación Física son muy divertidas, el profesor pone juegos muy chulos y además casi siempre el juega con nosotros» (EA).

Aunque en las clases existe un clima favorable, en algunas ocasiones, sobre todo al principio del curso, se podía observar como los alumnos querían intervenir todos al mismo tiempo, y hablaban todos a la vez. Eso conllevaba a que nadie escuchaba a nadie y el profesor no podía responder a ninguna duda de los alumnos.

«Existe respeto entre ellos aunque desde el principio del curso he señalado la importancia de respetar el turno de palabra». (EP).

A medida que pasaban las sesiones los alumnos y alumnas iban respetando el turno de palabra. Por ello, poco a poco, tanto en la parte inicial de la sesión como en la vuelta a la calma y la recogida de material el ambiente iba siendo cada vez más tranquilo. En las clases ya no hay mucho ruido $(\mathrm{O})$
Influencia del contexto educativo y social en el clima de aula.

En esta categoría vamos a estudiar cómo es la relación de las familias de los alumnos y alumnas del grupo clase con el centro educativo y concretamente con el profesor de dicho grupo. Comenzaremos diciendo que para el docente es importante que la familia esté apoyando y ayudando a sus hijos:

«Considero que la familia es muy importante en la educación de los niños, pues los alumnos/as hacen y dicen lo que ven en casa. Quiero tener un contacto directo con las familias para conocer el comportamiento de los niños». (EP).

En la mayoría de los casos las madres se preocupan por el comportamiento de sus hijos e hijas dentro de la escuela, y cuando vienen a recogerles suelen hablar con el profesorado $(\mathrm{O})$.

Como observador, cuando la clase de Educación Física era a última hora de la mañana, me quedaba a presenciar cómo era la relación del docente con los padres cuando estos venían a recoger a sus hijos. Se notaba que el clima era bueno, de colaboración, las madres realizaban preguntas interesándose por cómo se había portado su hijo hoy, por cómo iba en las clases, etc. El docente se mostraba amable y cómodo, dando explicaciones a las madres, y se hablaban de más cosas. Por ejemplo un día una madre trajo a su hijo de un año y el docente estuvo preguntándole por el y jugando con el niño.

"Conozco la situación familiar de cada alumno y alumna, como por ejemplo el número de hermanos que tiene cada uno.»(CP).

Respecto a sí las familias ayudan de verdad a sus hijos y si están pendientes de ellos y se preocupan por sus ellos, tenemos respuestas como:

«Se nota que en casa le ayudan con las tareas, y sus padres hablan mucho conmigo como tutor del curso que soy». (EP).

«Me ayudan en todo lo que yo no entiendo.»(EA)

Por todo ello, y como hemos podido comprobar durante la investigación, podemos afirmar que las familias se preocupan por la educación y formación que reciben sus hijos. $(\mathrm{O})$.

\section{Evaluación del proceso}

Durante esta investigación cualitativa ha sido imprescindible la interacción del investigador con el medio investigado, por ello es necesario establecer una serie de recursos para salvaguardar la veracidad de los datos obtenidos. Así, hemos tenido presente una serie de recursos para asegurar, fundamentar y evidenciar el valor del conocimiento generado por este tipo de estudios, los cuales han sido utilizado por autores como Guba (1981, citado en Gimeno \& Pérez, 1985), Santos (1990, 2000) y Pérez (1994):

- Validez interna o credibilidad: se trata de que el proceso de análisis refleje con precisión lo que sucede en la realidad. Para lograr la credibilidad de los datos, hemos prolongado la investigación durante un año académico utilizando la observación continua y persistente, se han realizado diversas formas de triangulación, y los informes resultantes han sido aprobados por agentes externos.

- Validez externa o transferibilidad: consiste en trasladar los resultados de la investigación a otros contextos, aunque esto siempre es limitado pues «cada contexto tiene su carácter concreto e irrepetibley de su estudio no se extrae la comprobación de hipótesis de carácter general». (Santos 1990, p.169).

- Confirmabilidad: se ha garantizado la neutralidad y objetividad de la investigación, pues todos los datos mostrados han sido aprobados por los participantes y hemos realizado una triangulación de métodos, fuentes y participantes.

- Dependencia: la investigación es consistente y fiable, pues garantizar la repetición de los resultados cuando se realicen investigaciones a los mismos participantes, en el mismo contexto, y usando las mismas técnicas para la investigación. Así, hemos dejado claro de qué forma se realizó la investigación, aportando descripciones minuciosas sobre el proceso seguido en el estudio, describiendo nuestra propia actitud y detallado las técnicas utilizadas. 


\section{Discusión: Consideraciones finales}

Se trata de unir los datos obtenidos en el informe de la investigación, con la documentación teórica existente en la bibliografía acerca del tema de estudio. Hemos de dejar claro que no son conclusiones finales, pues es difícil que si se generan conclusiones éstas puedan convertirse en criterios únicos y exclusivos a tener en cuenta para cualquier situación y momento. Pues hemos estudiado una realidad única y es el lector quién debe elaborar sus propias generalizaciones encontrando, a partir de nuestros asertos y descripción, las coincidencias con su caso particular. Las generalizaciones de la presente investigación las hemos dividido en tres apartados generales:

Actuación del docente: ha potenciado las relaciones entre alumnos y de éstos con el profesor, ha establecido con ellos relaciones cordiales, colaborativas y confiadas, y se ha mostrado cercano y disponible para los alumnos. Lo cual para Guil \& Mestre (1998), son actuaciones muy positivas para crear un adecuado y positivo clima de aula.

Además, desde el punto de vista de De Lucas (1993), ha realizado acciones muy positivas, pues ha mostrado un sincero interés hacia los alumnos, interactuando con ellos a partir de lo que ellos valoran. Tratando por igual a todos los alumnos y alumnas independientemente de su nivel, capacidad o destreza a la hora de realizar las acciones físicomotrices. Por ello, según Siedentop (1998), existe más relación entre el docente y los discentes. Además, el docente ha conseguido crear un clima donde los alumnos/as se impliquen en las tareas, así, según García $y$ cols. (2005), se puede mejorar y hacer más adaptativos los patrones conductuales de los alumnos.

Metodología utilizada por el docente: ha utilizado estilos de enseñaza participativos, lo que, para Santos (1990), hace que aumente la participación y responsabilidad de los discentes y con ello mejore la convivencia. En la mayoría de ocasiones uso un feedback o conocimiento de resultados positivo, mejorando así el clima de aula (Sáenz-López, 1997). Y ha empleado un sistema de organización eficaz, pues ha establecido una serie de rutinas, normas y reglas de funcionamiento y comportamiento para ser respetadas y seguidas por todo el grupo (docente y discentes) desde el principio hasta el final del curso. Siedentop (1998) apunta que con esas acciones se ve aumentado el respeto y la cooperación de los alumnos entres sí y con el profesor.

Actuación del alumno: se ha comprobado la correcta actuación del alumnado dado que uno de sus objetivos y fines dentro del centro educativo era la socialización con los demás alumnos. Esto desde el punto de vista de Siedentop (1998) es positivo puesto que existirá un clima de aula donde los discentes puedan divertirse haciendo actividades grupales. Esto, según palabras de Ascorra y cols. (2003), hace que exista un clima de clase con ausencia de violencia y de perturbaciones para el estudio y para la creación de buenos y nuevos amigos dentro de la propio grupo clase.

\section{Conclusiones}

Atendiendo a los datos aportados y basándonos en indicaciones propuestas por Posada (2000), proponemos una serie de orientaciones con el fin de mejorar el clima de aula dentro de las sesiones de Educación Física:

-Ofrecer al alumno una imagen de respeto y dignidad personal y profesional.

-Tratar al alumno con cariño y respeto interesándose por sus problemas.

-Mostrar actitudes positivas y de interés hacia la actividad física.

-Tener perspectivas positivas ante las actuaciones de los alumnos $\mathrm{y}$ alumnas.

-Establecer diálogos y debates sobre temas interesantes para los alumnos.

-Ofrecer credibilidad respetando las reglas y los compromisos adquiridos.

-Desprender buen humor y entusiasmo.
-Establecernormas de funcionamiento diario para mejorar la convivencia.

-Potenciar las relaciones sociales constructivas y equilibradas entre los alumnos y el docente y entre los alumnos entre sí.

\section{Referencias}

Ascorra, P., Arias, H. y Graff, C. (2003). La escuela como contexto de contención social y afectiva. Revista enfoques educacionales, vol. 5, $\mathrm{N}^{\mathrm{o}} 1$. EN:

http://www.csociales.uchile.cl/publicaciones/enfoques/07/ Ascorra Arias Graff EscuelaContencionSocialAfectiva.pdf. 06/ 11/06, 11:00h.

Gairín, J. (1986). Aprendizaje y cambio de actitud en la didáctica especial de las matemáticas. Tesis doctoral. Barcelona: Departamento de Pedagogía Aplicada. UAB.

García, T., Santos-Rosa, F., Jiménez, R., y Cervell, E. (2005). El clima motivacional en las clases de Educación Física: una aproximación práctica desde la Teoría de Metas de Logro. Apunts. Educación Física y Deportes, 81, 21-28.

Guba,E. (1981). Criterios de credibilidad en la investigación naturalista. En Gimeno, J. \& Pérez, A. (1985). La enseñanza: Su teoría y su práctica. Madrid: editorial Akal.

Guil, R. \& Mestre, J. (1998). Violencia escolar: su relación con las actitudes sociales del alumnado y el clima social del aula. Revista Electrónica Iberoamericana de Psicología Social.

EN: http://www.psico.uniovi.es/REIPS/v2n1/art1.html 6/11/06, 12:00 $\mathrm{h}$.

Gutiérrez, R. (1994). La estética del espacio escolar. Estudio de un caso. Tesis doctoral. Málaga: Secretariado de publicaciones de la Universidad de Málaga.

Gutiérrez, R. (2006). Los estudios de casos una opción metodológica para investigar la educación artística. En Martín, R. (2006). Investigación en educación artística. Granada: Universidades de Granaday de Sevilla.

Heras, L. (1994). Etnografia del espacio escolar. Estudio de un caso. Tesis doctoral. Málaga: Secretariado de publicaciones de la Universidad de Málaga.

Decreto 230/2007, de 31 de julio, por el que se establece la ordenación y las enseñanzas correspondientes a la educación primaria en Andalucía.

Martínez, M. (1996). La orientación del clima de aula. Investigación sobre el desarrollo de una investigación. Tesis doctoral. Barcelona: publicaciones de la Universidad Autónoma de Barcelona.

Pérez, G. (1994). Investigación cualitativa. Retos e interrogantes. II técnicas y análisis de datos. Madrid: editorial La Muralla.

Plaza, F. (1994). Análisis etnográfico de los determinantes de la disciplina en el centro escolar. Un estudio de casos. Tesis doctoral. Málaga: Secretariado de publicaciones de la Universidad de Málaga.

Posada, F. (2000). Ideas prácticas para la enseñanza de la Educación Física. Lérida: Agonós.

Prada, C. (1995). Relaciones de poder en la escuela. Estudio de un caso. Tesis doctoral. Málaga: Secretariado de publicaciones de la Universidad de Málaga.

Sáenz-López, P.(1997). Educación Física y su Didáctica. Manual para el profesor. Sevilla: Wanceulen editorial deportiva, S.L.

Santos, M. (1990). Hacer visible lo cotidiano. Teoría y práctica de la evaluación cualitativa de centros escolares. Madrid:Akal Universitaria.

Santos, M. (2000). Entre bastidores, el lado oculto de la organización escolar. Archidona:Aljibe.

Taylor, S. y Bogdan, R. (1986). Introducción a los métodos cualitativos de la investigación. La búsqueda de significados. Barcelona: Paidos.

Woods, P. (1995). La escuela por dentro: la etnografia en la investigación educativa. Barcelona: Paidos/MEC. 